\title{
Dark energy and the structure of the Coma cluster of galaxies
}

\author{
A. D. Chernin ${ }^{1,2}$, G. S. Bisnovatyi-Kogan ${ }^{3}$, P. Teerikorpi ${ }^{1}$, M. J. Valtonen ${ }^{1}$, G. G. Byrd ${ }^{4}$, and M. Merafina ${ }^{5}$ \\ 1 Tuorla Observatory, Department of Physics and Astronomy, University of Turku, 21500 Piikkiö, Finland \\ e-mail: pekkatee@utu.fi \\ 2 Sternberg Astronomical Institute, Moscow University, 119899 Moscow, Russia \\ 3 Space Research Institute, Russian Academy of Sciences, 117997 Moscow, Russia \\ ${ }^{4}$ University of Alabama, Tuscaloosa, AL 35487-0324, USA \\ 5 Department of Physics, University of Rome "La Sapienza", 00185 Rome, Italy
}

Received 23 November 2012 / Accepted 18 March 2013

\begin{abstract}
Context. We consider the Coma cluster of galaxies as a gravitationally bound physical system embedded in the perfectly uniform static dark energy background as implied by $\Lambda$ CDM cosmology.

Aims. We ask if the density of dark energy is high enough to affect the structure of a large and rich cluster of galaxies.

Methods. We base our work on recent observational data on the Coma cluster, and apply our theory of local dynamical effects of dark energy, including the zero-gravity radius $R_{\mathrm{ZG}}$ of the local force field as the key parameter.

Results. 1) Three masses are defined that characterize the structure of a regular cluster: the matter mass $M_{\mathrm{M}}$, the dark-energy effective mass $M_{\mathrm{DE}}(<0)$, and the gravitating mass $M_{\mathrm{G}}\left(=M_{\mathrm{M}}+M_{\mathrm{DE}}\right)$. 2) A new matter-density profile is suggested that reproduces the observational data well for the Coma cluster in the radius range from $1.4 \mathrm{Mpc}$ to $14 \mathrm{Mpc}$ and takes the dark energy background into account. 3) Using this profile, we calculate upper limits for the total size of the Coma cluster, $R \leq R_{\mathrm{ZG}} \approx 20 \mathrm{Mpc}$, and its total matter mass, $M_{\mathrm{M}} \lesssim M_{\mathrm{M}}\left(R_{\mathrm{ZG}}\right)=6.2 \times 10^{15} M_{\odot}$.

Conclusions. The dark energy antigravity affects the structure of the Coma cluster strongly at large radii $R \gtrsim 14 \mathrm{Mpc}$ and should be considered when its total mass is derived.
\end{abstract}

Key words. galaxies: clusters: individual: Coma - dark matter - dark energy

\section{Introduction}

The Coma cluster of galaxies (A1656) is the most massive wellstudied regular gravitationally bound aggregation of matter in the observable Universe. In his classic work Zwicky (1933, 1937) applied the virial theorem to the cluster and showed that dark matter dominates the system. Zwicky estimated its mass as $3 \times 10^{14} M_{\odot}$, when normalized to the Hubble constant $h=0.71$ used hereafter.

Decades later, The \& White (1986) found an order of magnitude higher value, $2 \times 10^{15} M_{\odot}$, with a generalization of the virial theorem that must be used when the observed sample does not include the entire system. Hughes $(1989,1998)$ obtained a similar value $(1-2) \times 10^{15} M_{\odot}$ with X-ray data under the assumption that the cluster's hot intergalactic gas is in hydrostatic equilibrium. In a similar way, Colles (2006) reports the mass $4.4 \times 10^{14} M_{\odot}$ inside the radius of $1.4 \mathrm{Mpc}$. A weak-lensing analysis gives $2.6 \times 10^{15} M_{\odot}$ (Kubo et al. 2007) within $4.8 \mathrm{Mpc}$ radius. Geller et al. $(1999,2011)$ extended mass estimates to the outskirts of the cluster using the caustic technique (Diaferio \& Geller 1997; Diaferio 1999) and find the mass $2.4 \times 10^{15} M_{\odot}$ within the $14 \mathrm{Mpc}$ radius. Here the $2 \sigma$ error is $1.2 \times 10^{15} M_{\odot}$, so it does not contradict the apparently higher mass within $4.8 \mathrm{Mpc}$.

In this paper, we re-examine the mass estimation of the Coma cluster using the data above and a new theory model that describes the cluster as a bound spherical system embedded in the cosmic background of dark energy. We find that dark energy strongly affects the cluster structure at large distances $R \geq 14 \mathrm{Mpc}$ from the cluster center and must be taken into account in the mass estimate.
Basic theory is outlined in Sect. 2, three characteristic masses of a regular cluster are introduced in Sect. 3, a new matter mass profile is defined in Sect. 4, the upper bound on the total size of the Coma cluster is calculated and discussed in Sect. 5, and the results are summarized in Sect. 6.

\section{Local dynamical effects of dark energy}

We adopt the $\Lambda$ CDM cosmology that identifies dark energy with Einstein's cosmological constant $\Lambda$ and treats it as a perfectly uniform vacuum-like fluid with the constant density $\rho_{\mathrm{DE}}=$ $0.71 \times 10^{-29} \mathrm{~g} \mathrm{~cm}^{-3}$. The dark energy background produces antigravity that is stronger than the matter gravity in the present Universe as a whole. This makes the cosmological expansion accelerate, as discovered by Riess et al. (1998) and Perlmutter et al. (1999).

The cosmic antigravity can be stronger than gravity not only globally, but also locally on scales of $\sim 1-10 \mathrm{Mpc}$ (Chernin et al. 2000, 2006; Chernin 2001; Byrd et al. 2007, 2012), as studied using the HST observations made by Karachentsev's team (e.g., Chernin et al. 2010, 2012a).

The local weak-field dynamical effects of dark energy can be adequately described in terms of Newtonian mechanics (e.g., Chernin 2008). Such an approach borrows from general relativity the major result: the effective gravitating density of a uniform medium is given by the sum

$\rho_{\mathrm{eff}}=\rho+3 P$, 
where $\rho$ and $P$ are the fluid's density and pressure ( $c=1$ hereafter). In the $\Lambda$ CDM model, the dark energy equation of state is $P_{\mathrm{DE}}=-\rho_{\mathrm{DE}}$, and its effective gravitating density

$\rho_{\mathrm{DE} e f f}=\rho_{\mathrm{DE}}+3 P_{\mathrm{DE}}=-2 \rho_{\mathrm{DE}}<0$

is negative, producing antigravity. Einstein's "law of universal antigravity" says that a point mass $M$ within uniform dark energy generates an acceleration $a(r)$ that includes, in addition to the Newtonian term $a_{\mathrm{N}}(r)=-G M / r^{2}$, the antigravity effect of dark energy

$a_{\mathrm{E}}(r)=-\frac{4 \pi G}{3} \rho_{\mathrm{DEeff}} r^{3} / r^{2}=+\frac{8 \pi G}{3} \rho_{\mathrm{DE}} r$.

Then a test particle at the distance $R$ from the center of a spherical matter mass $M_{\mathrm{M}}$ (beyond the mass) has the radial acceleration in the reference frame of the mass center:

$a(R)=a_{\mathrm{N}}(R)+a_{\mathrm{E}}(R)=-G \frac{M_{\mathrm{M}}}{R^{2}}+\frac{8 \pi G}{3} \rho_{\mathrm{DE}} R$.

Equation (4) comes from the Schwarzschild-de Sitter spacetime in the weak field approximation. The net acceleration $a(R)$ is zero at the distance (Chernin et al. 2000; Chernin 2001, 2008)

$R=R_{\mathrm{ZG}}=\left[\frac{M_{\mathrm{M}}}{\frac{8 \pi}{3} \rho_{\mathrm{DE}}}\right]^{1 / 3}=11\left(\frac{M_{\mathrm{M}}}{10^{15} M_{\odot}}\right)^{1 / 3} \mathrm{Mpc}$,

Gravity dominates at distances $R<R_{\mathrm{ZG}}$, while antigravity is stronger than gravity at $R>R_{\mathrm{ZG}}$. A gravitationally bound system with the mass $M_{\mathrm{M}}$ can only exist inside its zero-gravity sphere of the radius $R_{\mathrm{ZG}}$.

\section{Three masses of a regular cluster}

The presence of dark energy in the spherical volume of a regular cluster like Coma may be quantified by its effective gravitating mass within a given clustrocentric radius $R$ :

$M_{\mathrm{DE}}(R)=-\frac{8 \pi}{3} \rho_{\mathrm{DE}} R^{3}=-0.85 \times 10^{15}\left[\frac{R}{10 \mathrm{Mpc}}\right]^{3} M_{\odot}$.

The matter (dark matter and baryons) content of the cluster is characterized by the mass $M_{\mathrm{M}}(R)$ inside radius $R$ :

$M_{\mathrm{M}}(R)=4 \pi \int \rho(R) R^{2} \mathrm{~d} R$.

Here $\rho(R)$ is the matter density within the cluster. The sum

$M_{\mathrm{G}}(R)=M_{\mathrm{M}}(R)+M_{\mathrm{DE}}(R)$,

is the total gravitating mass within the radius $R$. It is this mass that can be directly measured by the methods cited in Sect. 1, which are all related to gravitation and give the gravitating mass $M_{\mathrm{G}}(R)$, rather than the matter mass $M_{\mathrm{M}}(R)$, which can, however, be derived from the data of Sect. 1 using Eq. (8): $M_{\mathrm{M}}=M_{\mathrm{G}}-M_{\mathrm{DE}}$. So one has for $R=1.4 \mathrm{Mpc}$ :

$M_{\mathrm{DE}}=-2.3 \times 10^{12} M_{\odot}, \quad M_{\mathrm{M}} \simeq M_{\mathrm{G}}=4.4 \times 10^{14} M_{\odot}$.

For $R=4.8 \mathrm{Mpc}$,

$M_{\mathrm{DE}}=-9.4 \times 10^{13} M_{\odot}, \quad M_{\mathrm{M}} \simeq M_{\mathrm{G}}=2.6 \times 10^{15} M_{\odot}$.

And for $R=14 \mathrm{Mpc}$,

$M_{\mathrm{DE}}=-2.3 \times 10^{15} M_{\odot}, \quad M_{\mathrm{M}}=4.7 \times 10^{15} M_{\odot}$.
As we see, in the inner cluster at $R=1.4$ and $4.8 \mathrm{Mpc}$, the dark energy contributes practically nothing compared to the gravitating mass, so $M_{\mathrm{G}} \simeq M_{\mathrm{M}}$ here. But (curiously) the absolute value of the dark energy mass $M_{\mathrm{DE}}$ nearly equals the gravitating mass $M_{\mathrm{G}}$ at $R=14 \mathrm{Mpc}$; as a result, the matter mass $M_{\mathrm{M}} \simeq 2 M_{\mathrm{G}} \simeq 2\left|M_{\mathrm{DE}}\right|$ at this radius. The difference between the Eq. (11) estimate and the observed value of $M_{\mathrm{G}}$ is at the level of $4 \sigma\left(1 \sigma \approx \frac{1}{4} M_{\mathrm{G}}\right.$, Sect. 1$)$.

In the outer regions of the Coma cluster,

$\left|M_{\mathrm{DE}}\right|>M_{\mathrm{G}}, \quad R>14 \mathrm{Mpc}$,

where the antigravity effect is thus significant.

\section{Matter mass profile}

Our estimate of the Coma matter mass within $R=14 \mathrm{Mpc}$ (Eq. (11)) may be compared with estimates following from traditional matter density profiles for dark halos.

\subsection{NFW and Hernquist profiles}

The popular NFW profile (Navarro et al. 2005) is

$\rho=\frac{4 \rho_{s}}{\frac{R}{R_{\mathrm{s}}}\left(1+\frac{R}{R_{\mathrm{s}}}\right)^{2}}$,

where $R$ is again the distance from the cluster center, $\rho_{s}=\rho\left(R_{\mathrm{S}}\right)$, and $R_{\mathrm{S}}$ are constant parameters. At small radii, $R \ll R_{\mathrm{s}}$, the matter density goes to infinity, $\rho \propto 1 / R$ as $R$ goes to zero. At long distances, $R \gg R_{\mathrm{s}}$, the density slope is $\rho \propto 1 / R^{3}$. With this profile, the matter mass profile is

$M_{\mathrm{M}}(R)=16 \pi \rho_{s} R_{\mathrm{s}}^{3}\left[\ln \left(1+R / R_{\mathrm{s}}\right)-\frac{R / R_{\mathrm{S}}}{1+R / R_{\mathrm{s}}}\right]$.

To find the parameters $\rho_{s}$ and $R_{\mathrm{S}}$, we use the small-radii data of Sect. $1: M_{1}=4.4 \times 10^{14} M_{\odot}$ at $R_{1}=1.4 \mathrm{Mpc}, M_{2}=2.6 \times 10^{15} M_{\odot}$ at $R_{2}=4.8 \mathrm{Mpc}$. At these radii, the gravitating masses are nearly equal to the matter masses there (cf. Sect. 3). The values of $M_{1}$, $R_{1}$ and $M_{2}, R_{2}$, together with Eq. (14) lead to two logarithmic equations for the two parameters of the profile, which can easily be solved: $R_{\mathrm{s}}=4.7 \mathrm{Mpc}, \rho_{s}=1.8 \times 10^{-28} \mathrm{~g} / \mathrm{cm}^{3}$. Then we find the matter mass within $R=14 \mathrm{Mpc}$,

$M_{\mathrm{M}} \simeq 8.7 \times 10^{15} M_{\odot}$,

to be considerably larger (over 70\%) than given by Eq. (11).

Another widely used density profile (Hernquist 1990) is

$\rho(R) \propto \frac{1}{R(R+\alpha)^{3}}$.

Its small-radius behavior is the same as in the NFW profile: $\rho \rightarrow$ $\infty$, as $R$ goes to zero. The slope at large radii is different: $\rho \propto$ $1 / R^{4}$. The corresponding mass profile is

$M_{\mathrm{M}}(R)=M_{0}\left[\frac{R}{R+\alpha}\right]^{2}$.

The parameters $M_{0}$ and $\alpha$ can be found from the same data as above on $M_{1}, R_{1}$ and $M_{2}, R_{2}: M_{0}=1.4 \times 10^{16} M_{\odot}, \quad \alpha=$ $6.4 \mathrm{Mpc}$, giving another value for the mass within $14 \mathrm{Mpc}$ :

$M_{\mathrm{M}}=6.6 \times 10^{15} M_{\odot}, \quad R=14 \mathrm{Mpc}$.

Now the difference from the figure of Eq. (11) is about $40 \%$. 


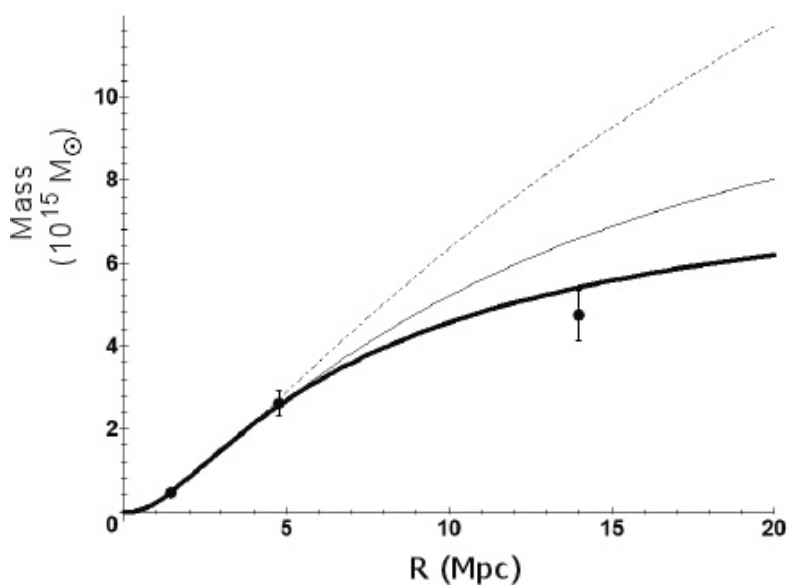

Fig. 1. Matter mass $M$ vs. radius $R$ for three profiles. 1) NFW mass profile of Eq. (14): $M=13.8\left[\ln (1+R / 4.7)-\frac{R}{4.7+R}\right]-$ dashed line; 2) Hernquist profile of Eq. (17): $M=14\left(\frac{R}{6.4+R}\right)^{2}-$ thin line; 3) New mass profile of Eq. (19): $M=8.7\left(\frac{R}{2.4+R}\right)^{3}-$ thick line. Here $R$ is in $\mathrm{Mpc}$ and $M$ is in $10^{15} M_{\odot}$. The values of the matter mass at radii $R=1.4,4.8,14 \mathrm{Mpc}$ are also shown as given by Eqs. (9)-(11) with $1 \sigma$ error bars.

\subsection{A modified mass profile}

In a search for a more suitable mass profile for the Coma cluster, we try a modified version of Hernquist's relation:

$M_{\mathrm{M}}(R)=M_{*}\left[\frac{R}{R+R_{*}}\right]^{3}$.

The power of three is used now instead of the power of two in Eq. (17). This mass profile comes from the density profile:

$\rho=\frac{3}{4 \pi} M_{*} R_{*}\left(R+R_{*}\right)^{-4}$

The density goes to a constant as $R$ goes to zero; at large radii, $\rho \propto 1 / R^{4}$, as in Hernquist's profile.

The parameters $M_{*}$ and $R_{*}$ are found again from the data for the radii of 1.4 and $4.8 \mathrm{Mpc}: M_{*}=8.7 \times 10^{15} M_{\odot}, \quad R_{*}=$ $2.4 \mathrm{Mpc}$. The new profile leads to a lower matter mass at $14 \mathrm{Mpc}$ :

$M_{\mathrm{M}}=5.4 \times 10^{15} M_{\odot}$,

which is equal to the Eq. (11) value within $15 \%$ accuracy. The three profiles can be found in Figs. 1 and 2.

\section{Discussion}

We now discuss some implications of the above results.

\subsection{Upper limits to size and mass}

The strong effect of dark energy at large radii puts an absolute upper limit on the total size of the cluster. The system can be gravitationally bound only if gravity dominates in its volume (as we mentioned in Sect. 2). In terms of the three different masses, this criterion may be given in the form

$M_{\mathrm{G}} \geq 0, \quad M_{\mathrm{M}} \geq\left|M_{\mathrm{DE}}\right|$.

Both inequalities are met, if the system is not larger than its zerogravity radius (Eq. (5)): $R \leq R_{\max }=R_{\mathrm{ZG}}$.

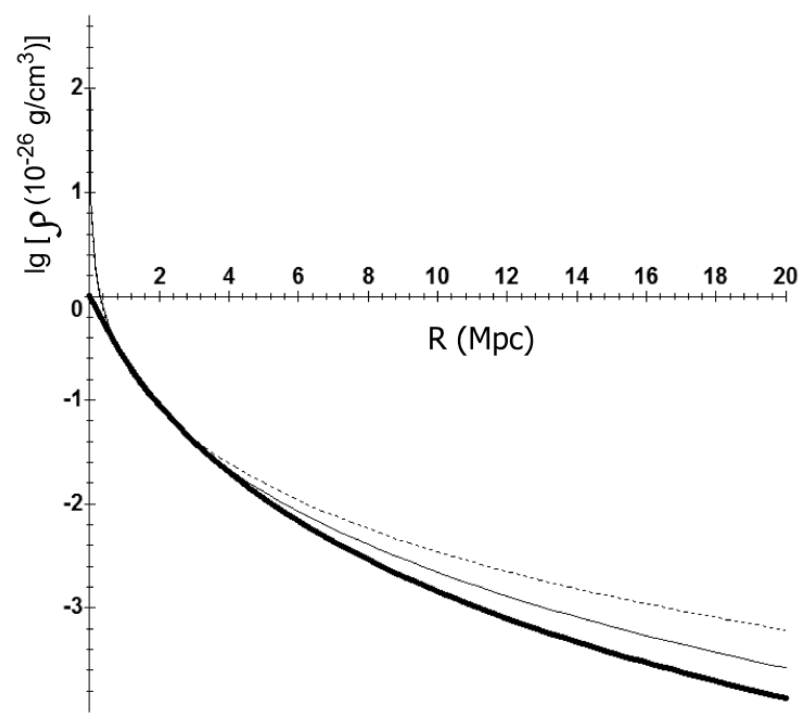

Fig. 2. Matter density $\rho$ vs. radius $R$. 1) NFW density profile (Eq. (13)): $\rho=\frac{7.5}{R(4.7+R)^{2}}-$ dashed line; 2) Hernquist profile (Eq. (16)): $\rho=$ $\frac{97}{R(6.4+R)^{3}}-$ thin line; 3) New density profile (Eq. (20)): $\rho=\frac{34}{(2.4+R)^{4}}-$ thick line.

If the radius of a system with matter mass $M_{\mathrm{M}}$ is equal to the maximal radius $R=R_{\max }$, its mean matter density (see Bisnovatyi-Kogan \& Chernin 2012) is

$\left\langle\rho_{\mathrm{M}}\right\rangle=\frac{M_{\mathrm{M}}}{\frac{4 \pi}{3} R_{\mathrm{ZG}}^{3}}=2 \rho_{\mathrm{DE}}$

This relation and the new profile (Eq. (19)) now lead to $R_{\max }$ and the corresponding matter mass, $M_{\max }=M_{\mathrm{M}}\left(R_{\max }\right)$ :

$R_{\max }=R_{\mathrm{ZG}}=20 \mathrm{Mpc}, M_{\max }=M_{\mathrm{M}}\left(R_{\mathrm{ZG}}\right)=6.2 \times 10^{15} M_{\odot}$.

For comparison, the other profiles lead to

$R_{\max }=25 \mathrm{Mpc}, M_{\max }=1.5 \times 10^{16} M_{\odot}(\mathrm{NFW})$,

$R_{\max }=22 \mathrm{Mpc}, M_{\max }=9.1 \times 10^{15} M_{\odot}$ (Hernquist).

\subsection{Close to the maximal size?}

Our studies of nearby systems like the Local Group and the Virgo and Fornax clusters (e.g., Chernin et al. 2010, 2012a) suggest their sizes are not far from the zero-gravity radii. Around them, flows of galaxies are seen (Karachentsev et al. 2009; Nasonova et al. 2011), the systems are located in the gravitydominated regions $\left(R<R_{\mathrm{ZG}}\right)$, and the outflows are at $R>R_{\mathrm{ZG}}$. If the local systems have nearly maximal sizes, this may explain the apparent underdensity of the local universe (Chernin et al. 2012b).

It is tempting to ask if the matter distribution could extend to somewhere near the maximal distance of $20 \mathrm{Mpc}$ in the Coma cluster as well. If so, its mass would be near the upper limit evaluated above, and still consistent with the theory of large-scale structure formation that claims the range $2 \times 10^{15}<M<10^{16} M_{\odot}$ for the most massive bound objects in the Universe (Holz \& Perlmutter 2012; Busha et al. 2005). Another implication is the predicted (Eq. (23)) mean matter density of the system = twice the dark energy density, which does not depend on the density profile assumed (Merafina et al. 2012; Bisnovatyi-Kogan \& Chernin 2012). Its observational confirmation would directly indicate the key role of dark energy for the 
structure of the system. Using the cosmological matter density parameter $\Omega_{\mathrm{m}}=0.27$, its mean density contrast would be

$\delta=\frac{\langle\rho\rangle-\rho_{\mathrm{m}}}{\rho_{\mathrm{m}}}=\frac{2 \Omega_{\mathrm{DE}}}{\Omega_{\mathrm{m}}}-1=4.2$.

Another general prediction (Chernin et al. 2006) is that at distances $R>R_{\mathrm{ZG}}$ any galaxy in the outflow should have a velocity higher than

$V_{\mathrm{esc}}=H_{\Lambda} R\left[1+2\left(R_{\mathrm{ZG}} / R\right)^{3}-3\left(R_{\mathrm{ZG}} / R\right)^{2}\right]^{1 / 2}$.

Here $H_{\Lambda}=\left[\frac{8 \pi G}{3} \rho_{\mathrm{DE}}\right]^{1 / 2}=61 \mathrm{kms}^{-1} \mathrm{Mpc}^{-1}$ depends on the dark energy density alone. Furthermore, within the simplified model of the Einstein-Straus vacuole, one expects the flow to reach the global Hubble rate at the edge of the vacuole $\left(\approx 1.7 R_{\mathrm{ZG}}\right.$, Teerikorpi \& Chernin 2010; Hartwick 2011).

The situation is complicated by the fact that the Coma cluster is not isolated, but lies within the CfA Great Wall.

\subsection{Dark energy estimator}

We have assumed that the dark energy density inside the Coma cluster is equal to its global value. One can reverse the argument and consider the local dark energy density as an unknown constant $\rho_{x}$. Its value may be independently estimated using our concept of three cluster masses.

The data (Geller et al. 1999, 2011) give the gravitating mass $M_{\mathrm{G}}$ within $R=14 \mathrm{Mpc}$. The mass $M_{\mathrm{M}}$ at the same radius may be found by extrapolating the data from $R=1.4 \mathrm{Mpc}$ and $R=4.8 \mathrm{Mpc}$ using a reasonable matter density profile (Sect. 4). Then with the masses $M_{\mathrm{G}}$ and $M_{\mathrm{M}}$ known for $R=14 \mathrm{Mpc}$, we may find the dark energy mass, $M_{\mathrm{DE}}(R)=M_{\mathrm{G}}-M_{\mathrm{M}}$, at the same radius. Finally, the dark energy density inside the cluster is estimated from

$\rho_{x}=\left|M_{\mathrm{G}}-M_{\mathrm{M}}\right| \frac{1}{\frac{8 \pi}{3} R^{3}} ; \quad R=14 \mathrm{Mpc}$.

With the density profiles of Eqs. (13) and (16) in Sect. 4, Eq. (29) gives for the local dark energy density the values

$\rho_{x}=(1.2-2) \times 10^{-29} \mathrm{~g} \mathrm{~cm}^{-3}$,

equal to the global value $\rho_{\mathrm{DE}}$ within an order-of-magnitude accuracy. (To avoid a circular argument, we do not use the profile of Eq. (20) here, as was partly suggested from considerations related to the global value itself.)

\section{Conclusions}

Three masses that characterize the structure of a regular cluster (like Coma) are defined as functions of the radius $R$ : the matter (dark matter and baryons) mass $M_{\mathrm{M}}(R)$, the dark-energy effective gravitating mass $M_{\mathrm{DE}}$ (negative), and the total gravitating mass $M_{\mathrm{G}}(R)=M_{\mathrm{M}}+M_{\mathrm{DE}}$. Of these masses, only the gravitating mass $M_{\mathrm{G}}$ reveals itself directly in observations at various distances from the cluster center. The dark energy mass $M_{\mathrm{DE}}$ may be derived using the known global value of the dark energy density. The mass $2.4 \times 10^{15} M_{\odot}$ measured at $R=14 \mathrm{Mpc}$ by Geller et al. $(1999,2011)$ is the gravitating mass $M_{\mathrm{G}}$ inside this radius. The corresponding matter mass is $M_{\mathrm{M}} \simeq 2 M_{\mathrm{G}}$.
At small radii, $R \ll 14 \mathrm{Mpc}$, dark energy effects are almost negligible, $\left|M_{\mathrm{DE}}\right| \ll M_{\mathrm{M}}$, and the gravitating mass $M_{\mathrm{G}}$ is practically equal to the matter mass $M_{\mathrm{M}}$. At large radii $R \geq 14 \mathrm{Mpc}$, the antigravity effects are strong and $\left|M_{\mathrm{DE}}\right| \geq M_{\mathrm{G}}$.

A new matter-mass profile for the Coma cluster reproduces the observational data well and accounts for the dark energy effects in the radius range from 1.4 to $14 \mathrm{Mpc}$ and beyond: $M_{\mathrm{M}}(R)=M_{*}\left[\frac{R}{R+R_{*}}\right]^{3}$, where the constants $M_{*}$ and $R_{*}$ can be found from the data for small radii.

The available observational data and the new mass profile give upper limits for the Coma cluster total size, $R \lesssim 20 \mathrm{Mpc}$, and total matter mass, $M_{\mathrm{M}} \lesssim 6.2 \times 10^{15} M_{\odot}$.

Acknowledgements. We thank Yu. N. Efremov, I. D. Karachentsev, D. I. Makarov, O. G. Nasonova, and A. V. Zasov for many useful discussions. A.C. appreciates partial support from the RFBR grant 13-02-00137. The work of G.B.-K. was partly supported by RFBR grant 11-02-00602, the RAN Program "Formation and evolution of stars and galaxies", and Russian Federation President Grant for Support of Leading Scientific Schools NSh-3458.2010.2. We also thank the anonymous referee for useful comments.

\section{References}

Bisnovatyi-Kogan, G. S., \& Chernin, A. D. 2012, Ap\&SS, 338, 337

Busha, M. T., Evrard, A. E., Adams, F. C., \& Wechsler, R. H. 2005, MNRAS, 363, L11

Byrd, G. G., Chernin, A. D., \& Valtonen, M. J. 2007, Cosmology: Foundations and Frontiers, Moscow, URSS

Byrd, G. G., Chernin, A. D., Teerikorpi, P., \& Valtonen, M. J. 2012, Paths to Dark Energy: Theory and Observation (Berlin: de Gruyter)

Chernin, A. D. 2001, Physics-Uspekhi, 44, 1099

Chernin, A. D. 2008, Physics-Uspekhi, 51, 267

Chernin A. D., Teerikorpi, P., \& Baryshev, Yu. V. 2000, Adv. Space Res., 31, 459, 2003

Chernin, A. D., Teerikorpi, P., \& Baryshev, Yu. V. 2006, A\&A, 456, 13

Chernin, A. D., Karachentsev, I. D., Nasonova, O. G., et al. 2010, A\&A, 520, A104

Chernin, A. D., Teerikorpi, P., Valtonen, M. J., et al. 2012a, Astron. Rep., 56, 653

Chernin, A. D., Teerikorpi, P., Valtonen, M. J., et al. 2012b, A\&A, 539, A4

Colles, M. 2006, in Encyclopedia of Astronomy and Astrophysics, ed. P. Murdin, IOP Pubs. Ltd, UK, 245

Diaferio, A. 1999, MNRAS, 309, 610

Diaferio, A., \& Geller, M. J. 1997, ApJ, 481, 633

Geller, M. J., Diaferio, A., \& Kurtz, M. J. 1999, ApJ, 517, L23

Geller, M. J., Diaferio, A., \& Kurtz, M. J. 2011, AJ, 142, 143

Hartwick, F. D. A. 2011, AJ, 141, 198

Hernquist, L. 1990, ApJ, 356, 359

Holz, D. E., \& Perlmutter, S. 2012, ApJ, 755, L36

Hughes, J. P. 1989, ApJ, 337, 21

Hughes, J. P. 1998, Untangling Coma Berenices: A New Vision of an Old Cluster, Proc. meeting held in Marseilles, June 17-20, 1997, eds. A. Mazure, F. Casoli, F. Durret, \& D. Gerbal, Word Scientific, 175

Karachentsev, I. D., Kashibadze, O. G., Makarov, D. I., et al. 2009, MNRAS, 393, 1265

Kubo, J. M., Stebbins, A., Annis, J., et al. 2007, ApJ, 671, 1466

Merafina, M., Bisnovatyi-Kogan, G. S., \& Tarasov, S. O. 2012, A\&A, 541, A84

Nasonova, O. G., de Freitas Pacheco, J. A., \& Karachentsev, I. D. 2011, A\&A, 532, A104

Navarro, J., Frenk, C. S., \& White, S. D. M. 2005, ApJ, 463, 563

Perlmutter, S., Aldering, G., Goldhaber, G., et al. 1999, ApJ, 517, 565

Riess, A. G., Filippenko, A. V., Challis, P., et al. 1998, AJ, 116, 1009

Rines, K., \& Diaferio, A. 2006, AJ, 132, 1275

Rines, K., Geller, M. J., Diaferio, A., Kurtz, M. J., \& Jarrett, T. H. 2004, AJ, 128, 1078

Teerikorpi, P., \& Chernin, A. D. 2010, A\&A, 516, A93

The, L. S., \& White, S. D. M. 1986, AJ, 92, 1248

Zwicky, F. 1933, Helvetica Phys. Acta, 6, 110

Zwicky, F. 1937, ApJ, 86, 217 